\section{A Characterization of the expression of 14-3-3 isoforms in psoriasis, basal cell carcinoma, atopic dermatitis and contact dermatitis}

\author{
Line Raaby, Kristian Otkjær, \\ Maria Luise Salvskov-Iversen, \\ Claus Johansen, Lars Iversen \\ Department of Dermatology, Aarhus \\ University Hospital, Aarhus, Denmark
}

\section{Abstract}

14-3-3 is a highly conserved protein involved in a number of cellular processes including cell signalling, cell cycle regulation and gene transcription. Seven isoforms of the protein have been identified; $\beta, \gamma, \varepsilon, \zeta, \eta, \sigma$ and $\tau$. The expression profile of the various isoforms in skin diseases is unknown. To investigate the expression of the seven 14-3-3 isoforms in involved and uninvolved skin from psoriasis, basal cell carcinoma (BCC), atopic dermatitis and nickel induced allergic contact dermatitis. Punch biopsies from involved and uninvolved skin were analyzed with quantitative reverse transcription-polymerase chain reaction to determine the mRNA expression of the 14-3-3 isoforms. The protein level of 14-3-3 isoforms was measured by Western blot technique in keratome biopsies from patients with psoriasis. Evaluation of dermal and epidermal protein expression was performed by immunofluorescence staining. Increased 14-3-3 $\tau$ mRNA levels were detected in involved skin from patients with psoriasis, contact dermatitis and BCC. 14-3-3 $\sigma$ mRNA expression was increased in psoriasis and contact dermatitis, but not in BCC. In atopic dermatitis no significant difference between involved and uninvolved skin was found. The expression of the 14-3-3 isoforms was also studied at the protein level in

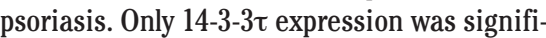
cantly increased in involved psoriatic skin compared with uninvolved skin. Immuno-

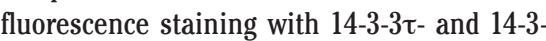
$3 \sigma$-specific antibodies showed localization of both isoforms to the cytoplasm of the keratinocytes in the various skin sections. These results demonstrate a disease specific expression profile of the $14-3-3 \tau$ and $14-3-3 \sigma$ isoforms.

\section{Introduction}

14-3-3 is a highly conserved protein family comprising seven different isoforms with molecular weights of 29-33 kDa., ${ }^{1,2}$ The 14-3-3 protein was first discovered in 1967 by Moore and Perez as an abundant brain protein ${ }^{3}$ and it has been found in all eukaryote organisms investigated so far . ${ }^{4} 14-3-3$ is found in all tissues $^{5}$ and interacts with more than 200 proteins. ${ }^{6}$ It is involved in a number of cellular processes including cell signalling, regulation of the cell cycle, intracellular trafficking, regulation of the cytoskeletal structure and control of gene transcription. ${ }^{2}$ In general the cellular effects of 14-3-3 can be classified into three categories $^{7}$ : i) Directing cell conformational changes; ii) binding to target proteins and modulation of their activity and/or interactions with other cellular components; iii) acting as a phosphorylation-dependent scaffold protein that recruits other proteins. Seven different isoforms of 14-3-3 have been identified and are denominated $\sigma, \zeta, \eta, \gamma, \tau, \varepsilon$ and $\beta$. Further more, $\alpha$ and $\delta$ isoforms have been identified as phosphorylated forms of $\beta$ and $\zeta$, respectively. ${ }^{2,8}$ The functions of the different isoforms are not yet clarified. 14-3-3 $\sigma$ is the best characterized isoform and is in particular expressed in epithelial cells. ${ }^{9}$ The expression of 14-3-3o increases in the cells after DNA damage and together with p53 it plays an important role for the G2/M checkpoint in the cell cycle. ${ }^{10}$ The gene can be inactivated by $\mathrm{CpG}$ methylation (epigenetic silencing) of the promoter region, which is seen in different types of carcinomas, including basal cell carcinoma. ${ }^{9,11}$ The 14-3-3 $\tau$ isoform is important in embryogenesis and both knockout mice and Xenopus with inactivated $14-3-3 \tau$ do not survive embryonic development. ${ }^{12,13} 14-3-3 \zeta$ downregulates the level of p53 and is upregulated in more than $40 \%$ of advanced breast cancers. ${ }^{14} 14-3-3 \gamma$ and $14-3-3 \beta$ also seem to have oncogenic potential when overexpressed. Overexpression of 14-3-3 $\beta$ in NIH 3T3 cells stimulate cell growth and when injected in nude mice, it augments tumor formation, ${ }^{15}$ while overexpression of $14-3-3 \gamma$ in H322 lung cancer cells results in abnormal DNA replication and polyploidization. ${ }^{16}$ The expression of $14-3-3 \varepsilon$ is upregulated in aged skin compared with young skin, and the protein is induced and accumulated after ultraviolet radiation. The expression is higher in sunexposed skin compared with sun-protected skin from the same patient. ${ }^{17}$ Taken together, these findings demonstrate distinct properties of the various 14-3-3 isoforms.

All seven isoforms have been identified in human keratinocytes in vitro, whereas all isoforms except 14-3-3 $\sigma$ were found in fibroblasts. ${ }^{18}$ Western blot analysis of intact human epidermis and immunohistochemical staining of human skin sections detected six isoforms but not the $14-3-3 \tau .^{18}$ Although the 14-3-3 protein is expressed in human skin the role of 143-3 proteins in inflammatory skin diseases has only been sparsely investigated. Using
Correspondence: Claus Johansen, Dept. of Dermatology, Aarhus University Hospital, P.P. Ørumsgade 11 DK-8000 Aarhus C, Denmark.

E-mail: claus.johansen@ki.au.dk

Key words: 14-3-3 proteins, psoriasis, basal cell carcinoma, allergic contact dermatitis, atopic dermatitis.

Contributions: LR has done all experimental work (except from growing the cell cultures) and analysed the results. Has also taken part in the design of the study and collecting biopsies; KO has taken part in the design of the study and collecting biopsies, has supervised the experimental work, especially the part concerning qRT-PCR; MLS-I collecting biopsies and has grown the cell cultures; $\mathrm{CJ}$, taken part in the design of the study and selecting the methods, supervised the experimental work and the statistical analysis; LI has designed the study and been the main supervisor of the project.

Acknowledgments: this work was supported by the Novo Nordisk Foundation, Lundbeck Foundation, the Andreassens and Hougaards Foundation, Master cabinetmaker Sophus Jacobsen and Wife Astrid Jacobsen's Fond, the Danish Research Agency.

Conflicts of interest: the authors have no conflict of interest to disclose.

Received for publication: 28 September 2010. Accepted for publication: 8 October 2010 .

This work is licensed under a Creative Commons Attribution 3.0 License (by-nc 3.0).

CCopyright L. Raaby et al., 2010 Licensee PAGEPress, Italy

Dermatology Reports 2010; 2:e14 doi:10.4081/dr.2010.e14

immunohistochemistry and polymerase chain reaction (PCR) technique Lodygin et al. found similar 14-3-3 $\sigma$ expression level in both involved psoriatic skin and normal skin,

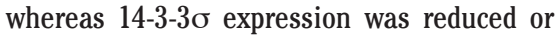
absent in basal cell carcinoma. ${ }^{11}$

The role of 14-3-3 in different common skin diseases is therefore unclear. The aim of this study was to characterize the mRNA profile of the seven 14-3-3 isoforms in four common skin diseases; i) psoriasis, a benign, Th-1 and Th-17 driven chronic inflammatory, hyperproliferating skin disorder; ii) atopic dermatitis, a Th-2 driven chronic inflammatory skin disease; iii) acute, allergic nickel induced contact dermatitis, a Th- 1 and Th-2 dominated disease ${ }^{19}$ and iv) basal cell carcinoma (BCC), a malignant hyperproliferating skin disorder. The protein profile of the seven 14-3-3 isoforms was only characterized in psoriatic skin because this was the only disease where sufficient tissue material could be collected allowing protein determination. 


\section{Materials and Methods}

\section{Biopsies}

BCC biopsies were taken from patients with histologically verified superficial or nodular BCC. The biopsies were taken from the center of the carcinoma. Biopsies from uninvolved skin were taken from normal skin from the same body region. From patients with atopic dermatitis punch biopsies from involved and uninvolved skin were taken from the same body region. The patients did not receive any systemic therapy. To investigate allergic contact dermatitis, skin biopsies from positive nickel patch test were used. The nickel patches were attached for 48 hours and the biopsies were taken 24 hours after the patch was removed. Uninvolved biopsies were taken from the same body region. The psoriatic skin biopsies were taken from patients with moderate to severe plaque psoriasis. The patients did not receive any systemic therapy for four weeks and no topical treatment for two weeks prior to the procedure. The involved skin biopsies were taken from the center of the plaque and the uninvolved skin biopsies were collected from the same body region. For quantitative reverse transcription-polymerase chain reaction (qRTPCR) analysis and immunofluorescence staining, $4 \mathrm{~mm}$ punch biopsies were taken while keratome biopsies were used for Western blotting. Biopsies for Western blotting and qRTPCR were immediately snap-frozen in liquid nitrogen, whereas biopsies for immunofluorescence staining were embedded in paraffin.

The study was conducted according to the Declaration of Helsinki Principles. The medical ethical committee of Aarhus University Hospital approved the study. Informed, written consent was obtained from each patient.

\section{Cell cultures}

Normal adult human keratinocytes were obtained by trypsinization of skin samples from patients undergoing plastic surgery as previously described. ${ }^{20}$ Second passage cultures were grown in keratinocyte serum-free medium with supplement containing epidermal growth factor (EGF) human recombinant and bovine pituitary extract, and gentamicin (Gibco/Invitrogen, Carlsbad, CA, USA.) to approximately $80 \%$ confluency. Twenty-four hours before stimulation the medium was changed to keratinocyte serum free media without supplement, in which the cells were stimulated. For stimulation we used tumor necrosis factors alpha (TNF- $\alpha)\left(10 \mathrm{ng} \mathrm{mL}^{-1}\right)$, interferon-gamma (IFN- $\gamma)\left(10 \mathrm{ng} \mathrm{mL}^{-1}\right)$, interleukin-1beta (IL-1 $\beta)$ (1 ng mL $\mathrm{mL}^{-1}$ ) (R\&D systems, Abingdon, UK), anisomysin (300 ng $\left.\mathrm{mL}^{-1}\right)$ and 12-0-tetradecanonylphorbol-13acetat (TPA) (50 nM), (Sigma-Aldrich, Inc., St. Louis, MO, USA). Keratinocytes stimulated with $\mathrm{CaCl}_{2}(0.3 \mathrm{mM})$ was maintained in growth-supplemented medium throughout the experiment (Merck, Darmstadt, Germany).

\section{Western Blotting}

The protein extract was prepared from keratome biopsies. Biopsies were homogenized in a cell lysis buffer (20 mM Tris-base ( $\mathrm{pH} 7.5)$, $150 \mathrm{mM} \mathrm{NaCl}, 1 \mathrm{mM}$ EDTA, 1 \% Triton X-100, $2.5 \mathrm{mM}$ sodium pyrophosphate, $1 \mathrm{mM} \mathrm{Na} 3 \mathrm{V04}$, $1 \mu \mathrm{g} \mathrm{mL}^{-1}$ leupeptin and $1 \mathrm{mM}$ PMSF) and left on ice for 30 minutes. The samples were centrifuged at $10,000 \mathrm{xg}$ for 10 minutes at $4^{\circ} \mathrm{C}$, after which the supernatant constituted the cell lysate.

Equal amounts of protein (determined by Bradford) were separated by SDS-PAGE and blotted onto nitrocellulose membranes. The membranes were incubated with the following primary antibodies 14-3-3 $\beta$ (catalog no.

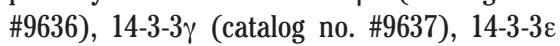
(catalog no. \#9635), 14-3-35 (catalog no.

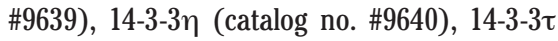
(catalog no. \#9638) from Cell Signaling Technology, Beverly, MA, USA, and 14-3-30 (catalog no. sc-100638) from Santa Cruz Biotechnology, Santa Cruz, CA, USA. Antibodies were detected with horseradish peroxidase-conjugated anti-rabbit (catalog no. \#7074 from Cell Signaling Technology, Beverly, MA, U.S.A.) or anti-mouse (catalog no. P0447, DAKO, Glostrup, Denmark) in a standard ECL reaction (GE Healthcare, Wessling, Germany). To estimate the protein size a biotinylated protein ladder molecular weight maker was used followed by anti-biotin (catalog no. \#7727 and \#7075 from Cell Signaling Technology, Beverly, MA, USA). Densitometric analysis of the band intensity was carried out using Kodak 1D Image Analysis Software.

\section{RNA isolation from biopsies}

Punch biopsies were transferred to $1 \mathrm{~mL}$ of $80^{\circ} \mathrm{C}$ cold RNAlater-ICE (Ambion Inc., Austin, TX, USA) and kept at $-80^{\circ} \mathrm{C}$. 24 hours before purification the biopsies were transferred to $-20^{\circ} \mathrm{C}$. Upon RNA purification, biopsies were removed from RNAlater-ice to $175 \mu \mathrm{L}$ SV RNA lysis buffer (added $\beta$-mercaptoethanol) and homogenized. RNA purification, including DNase treatment, was completed according to the manufacturer's instructions (SV Total RNA isolation Systems; Promega, Madison, WI, USA). The RNA was stored at $-80^{\circ} \mathrm{C}$ until further use.

\section{RNA isolation from cell cultures}

The cell supernatant was discarded and while on ice the keratinocytes were washed once in ice-cold sterile phosphate buffered saline (Gibco/Invitrogen, Carlsbad, CA, USA). $150 \mu \mathrm{L}$ RNA cell lysis buffer were added and the prepared lysate transferred to eppendorf tubes and kept at $-80^{\circ} \mathrm{C}$ until RNA purification. The purification was completed according to manufacturer's instructions (SV 96 Total RNA isolation Systems; Madison, WI, USA) RNA was stored at $-80^{\circ} \mathrm{C}$ until further use.

\section{Quantitative reverse transcription- polymerase chain reaction}

For reverse transcription TaqMan RT reagens (Applied Biosystems, Foster City, CA, USA) were used. Primers/probes for qRT-PCR were TaqmanGene expression Assay; (Assay ID: $14-3-3 \quad \beta, \quad H s 00268732 \_m 1 ; 14-3-3 \gamma$, Hs01113553_mH; 14-3-3ع, Hs00356749_g1; 14-

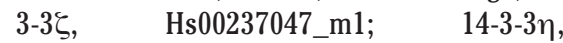
Hs00607046_ml; 14-3-3\%, Hs00356613_ml; 14-3-3 , Hs00863277_m1) (Applied Biosystems). As housekeeping gene RPLP0 was used (assay ID: Hs9999902_ml). The probes were FAM-labelled MGB with a nonflourescent quencher.

The expression of each gene was analysed in triplicate with $5 \mu \mathrm{L}$ of cDNA and $20 \mu \mathrm{L}$ PCR mastermix. The mastermix was Platinum ${ }^{\circledR}$ qPCR supermix-UDG (Invitrogen, Carlsbad, CA, U.S.A.). The real-time PCR machine was a Rotergene-3000 (Corbett Reseach, Sydney, Australia). Relative gene expression levels were determined using the relative standard curve method as outlined in User Bulletin no. 2 (ABI PRISM 7700 sequencing detection systems; Applied Biosystems). Briefly, a standard curve for each gene was made of 4-fold serial dilutions of total RNA from a punch biopsy from a psoriatic plaque. The curve was used to calculate relative amounts of mRNA in the samples. For 14-3-3 $\sigma$ the standard curve was made from RNA from normal adult human keratinocytes.

\section{Immunofluorescence}

The tissue sections were deparaffinized in xylene and hydrated through a descending ethanol series. For antigen unmasking, the sections were heated at $90^{\circ} \mathrm{C}$ for $15 \mathrm{~min}$. in a Tris/EGTA buffer (pH 9.0) followed by cooling for $20 \mathrm{~min}$. To block non-specific binding sites the sections were incubated with Image iT FX signal enhancer (Molecular Probes, Invitrogen, Carlsbad, CA, USA) for $30 \mathrm{~min}$. The sections were immunostained with 14-3-3 $\tau$ antibody (Cell Signaling Technology, Beverly, MA, USA

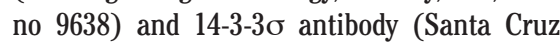
Biotechnology, no SC-100638) overnight at $4^{\circ} \mathrm{C}$. The next day the sections were incubated with secondary antibody (Alexa Flour 488 from Molecular Probes, Invitrogen) for 1 hour. Nuclear staining was performed by mounting samples in Prolong Gold antifade reagent with DAPI (Molecular Probes, Invitrogen). As negative control, sections were incubated with either blocking buffer without primary antibody or with normal rabbit IgG. 


\section{Statistical analysis}

For statistical analysis a Student's t-test (two-tailed) was preformed. $\mathrm{P}<0.05$ was regarded as statically significant. The results are expressed as mean \pm standard deviation.

\section{Results}

\section{4-3-3 mRNA expression profiles}

in psoriasis, atopic dermatitis, allergic contact dermatitis and basal cell carcinoma

To investigate the mRNA expression profile of the 14-3-3 family members in psoriasis, atopic dermatitis, allergic contact dermatitis, and basal cell carcinoma, paired punch biopsies were obtained from involved and uninvolved skin. Total RNA was isolated and

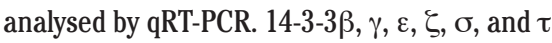
mRNA expression levels were significantly increased in involved psoriatic skin compared with uninvolved psoriatic skin $(n=9)$ (Figure $1 A)$. The 14-3-3 $\sigma$ and - $\tau$ mRNA levels were 2fold increased whereas 14-3-3 $\beta, \gamma, \varepsilon$ and $\zeta$ mRNA levels were less than 2-fold increased in involved psoriatic skin, compared with paired tissue samples of uninvolved psoriatic skin. No

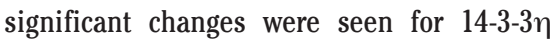
mRNA expression in psoriatic skin.

In biopsies from basal cell carcinomas $(\mathrm{n}=3)$ a significant 1.6-fold increase in 14-3-3ع mRNA expression was seen when compared with biopsies from normal skin from the patients (Figure 1B). 14-3-3 $\tau$ mRNA expression was increased 2.4 fold $(\mathrm{P}=0.052)$ in biopsies from basal cell carcinomas. No significant changes were seen in mRNA levels of any of the other isoforms.

Figure 1C shows the mRNA level of the various 14-3-3 isoforms in three patients with atopic dermatitis $(n=3)$. Although some variation in mRNA expression was found, no statistically significant differences were seen between involved and uninvolved atopic skin for any of the investigated 14-3-3 isoforms.

The results from three patients with nickelinduced contact dermatitis are displayed in figure 1D. Biopsies were collected 72 hours after patch testing with nickel. Analysis showed a significant 2.7-fold increase in 14-3-3 $\tau$ and $-\sigma$ mRNA expression in involved skin compared with uninvolved skin. 14-3-3 $\beta,-\gamma$ and $-\eta$ mRNA levels were also increased in involved skin, but the differences were not statistically significant.
14-3-3 protein expression in psoriatic skin

To assess 14-3-3 isoform protein expression levels by Western blotting technique, keratome biopsies from nine psoriasis patients were collected. It was not feasible to collect keratome biopsies from patients with BCC, atopic eczema or contact dermatitis. The protein expression in involved psoriatic skin was compared with

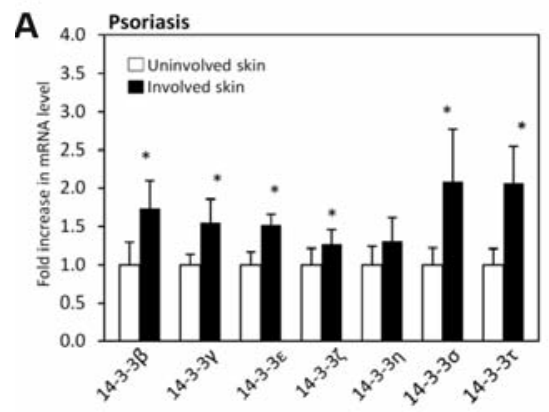

B 4.0 Basal cell carcinoma
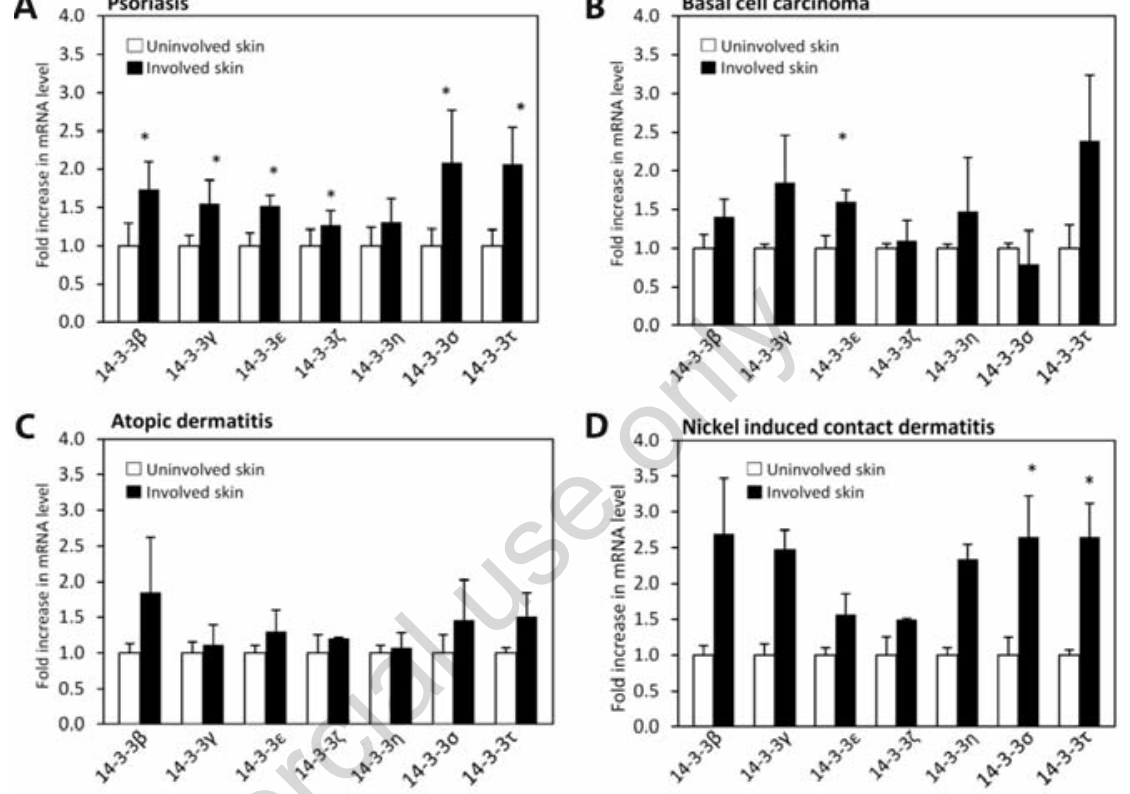

D

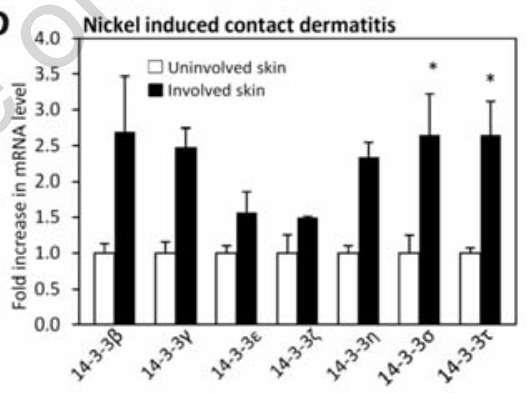

Figure 1. 14-3-3 mRNA expression in psoriasis (A), basal cell carcinoma (B), atopic dermatitis (C) and contact dermatitis (D). mRNA expression was analysed in paired punch biopsies from involved and uninvolved skin by quantitative reverse transcription-polymerase chain reaction. mRNA levels were normalized to the housekeeping gene RPLP0 and uninvolved biopsies were indexed to one. Bars indicate mean $\pm S D$. ${ }^{*} P<0.05$. ( $n=9$ for psoriasis, $\mathrm{n}=3$ for BCC, atopic dermatitis and contact dermatitis).
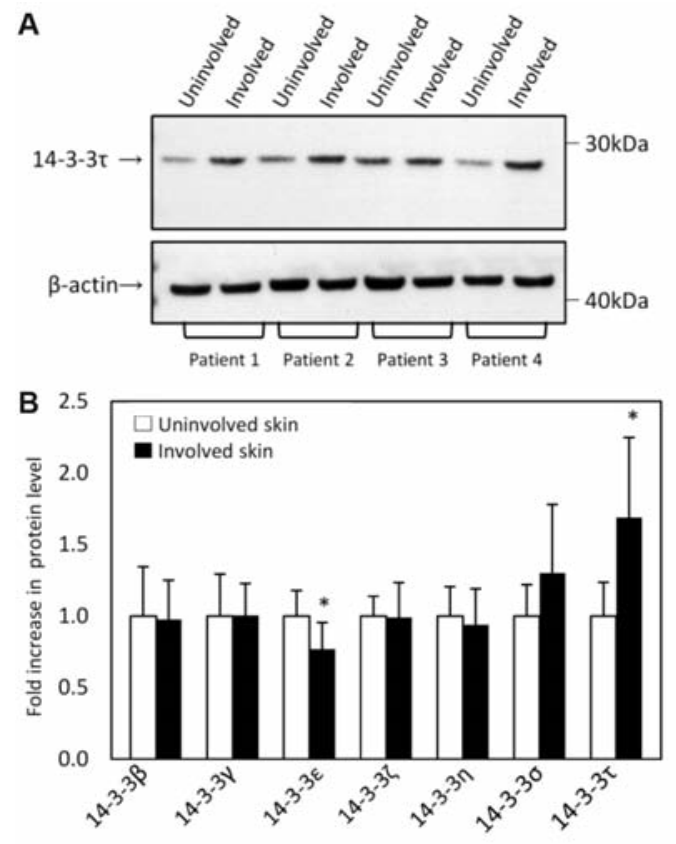

Figure 2. Increased level of $14-3-3 \tau$ protein in involved compared with uninvolved psoriatic skin. Protein extracts prepared from keratome biopsies taken from involved and uninvolved psoriatic skin were analysed with Western blot technique. (A) Shows the membrane probed with an antibody detecting $14-3-3 \tau$. $\beta$-actin was added to demonstrate equal protein loading. Pt. 1-4 is randomly chosen between the nine patients totally included. (B) Shows the protein level of all seven 14-3-3 isoforms. Band intensity was quantified using Kodak 1D Image Analysis Software. The protein level was normalized to $\beta$-actin and uninvolved biopsies were indexed to one. Bars indicate mean $\pm S D$. ${ }^{*} \mathbf{P}<\mathbf{0 . 0 5}$. 


\section{Immunofluorescence of 14-3-3} and $14-3-3 \sigma$

Because the mRNA expression of 14-3-3 $\tau$ and $14-3-3 \sigma$ was significantly upregulated by more than two fold in involved skin from both psoriasis and nickel induced contact dermatitis and a marked although statistically insignificant upregulation of $14-3-3 \tau$ was seen in BCC's, the localization of 14-3-3 $\tau$ and 14-3$3 \sigma$ was examined by immunofluorescence in involved and uninvolved tissue sections from

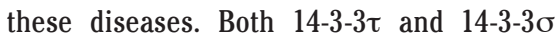
showed solid staining of the whole epidermis and higher magnification revealed localization of the proteins to the cytoplasm of the keratinocytes. The distribution of 14-3-3 $\tau$ (Figure $3 \mathrm{~A}-\mathrm{F}$ ) as well as $14-3-3 \sigma$ (Figure $4 \mathrm{~A}-\mathrm{F}$ ) was similar in involved and uninvolved skin in all investigated skin diseases.

\section{Regulation of 14-3-3 $\tau$ expression in cultured normal human keratino- cytes}

The mechanisms leading to increased 14-3$3 \tau$ mRNA expression in psoriasis, BCC, and allergic contact dermatitis are unknown. In an attempt to identify stimuli resulting in increased 14-3-3 $\tau$ mRNA expression, in vitro experiments were conducted with cultured normal human keratinocytes. Keratinocytes were stimulated with TNF- $\alpha$, IFN- $\gamma$, IL-1 $\beta$, TPA or anisomycin, respectively, for $3,6,12$, and 24 hours. Subsequently 14-3-3 $\tau$ mRNA levels were analyzed using qRT-PCR. No regulation of 14$3-3 \tau$ mRNA expression was seen for any of the stimuli investigated (Figure 5A). When keratinocyte differentiation was induced by calcium $(0.3 \mathrm{mM})$ the $14-3-3 \tau$ mRNA level increased. At 24 hours, 14-3-3 $\tau$ mRNA was increased 1.3-fold compared with medium controls $(\mathrm{P}<0.05)$. At 48 hours, increased calcium concentrations resulted in a 1.4 -fold increase in 14-3-3 $\tau$ mRNA. However, at this time point the increase was not statistically significant compared with medium controls (Figure 5B).

\section{Discussion}

14-3-3 isoforms are involved in the regulation of numerous cellular processes, but their expression in common skin disease has not previously been studied. The present study demonstrates a significant 2 -fold increase in the mRNA expression of $14-3-3 \tau$ and $-\sigma$ in involved psoriatic skin and in involved skin from nickel-induced allergic contact dermatitis compared with paired samples of uninvolved skin. In basal cell carcinomas only 14-3$3 \tau$ mRNA expression was increased more than 2-fold, whereas the 14-3-3 o mRNA level were slightly, although not significantly, decreased.
In involved and uninvolved atopic dermatitis skin no significant differences in the mRNA expression of the various isoforms could be detected. 14-3-3 protein expression was only studied in tissue samples from psoriasis patients and only the expression of the 14-3$3 \tau$ protein was significantly increased in involved psoriatic skin compared with uninvolved psoriatic skin.

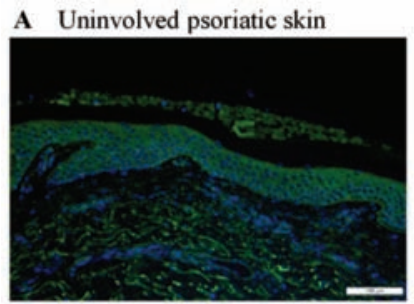

C Uninvolved BCC

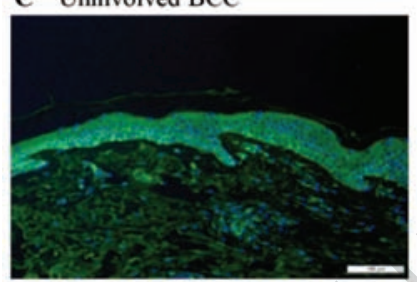

E Uninvolved contact dermatitis

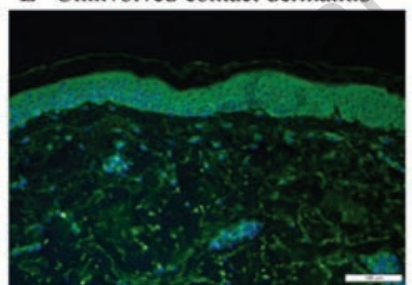

B Involved psoriatic skin

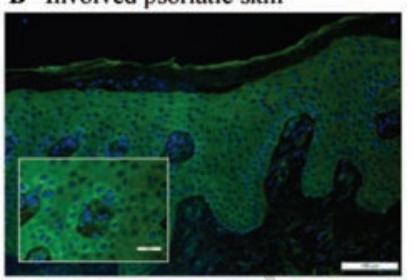

D Involved BCC

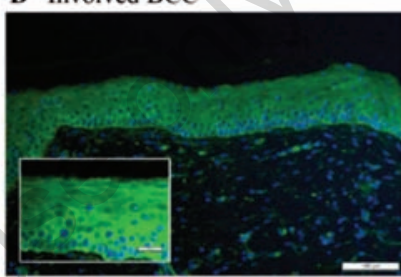

F Involved contact dermatitis

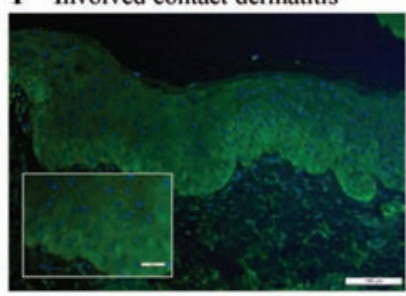

C Uninvolved BCC
A Uninvolved psoriatic skin

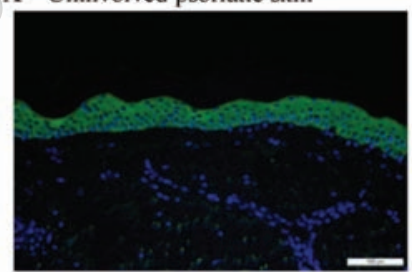

B Involved psoriatic skin

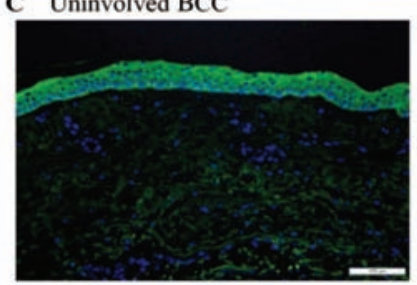

E Uninvolved contact dermatitis
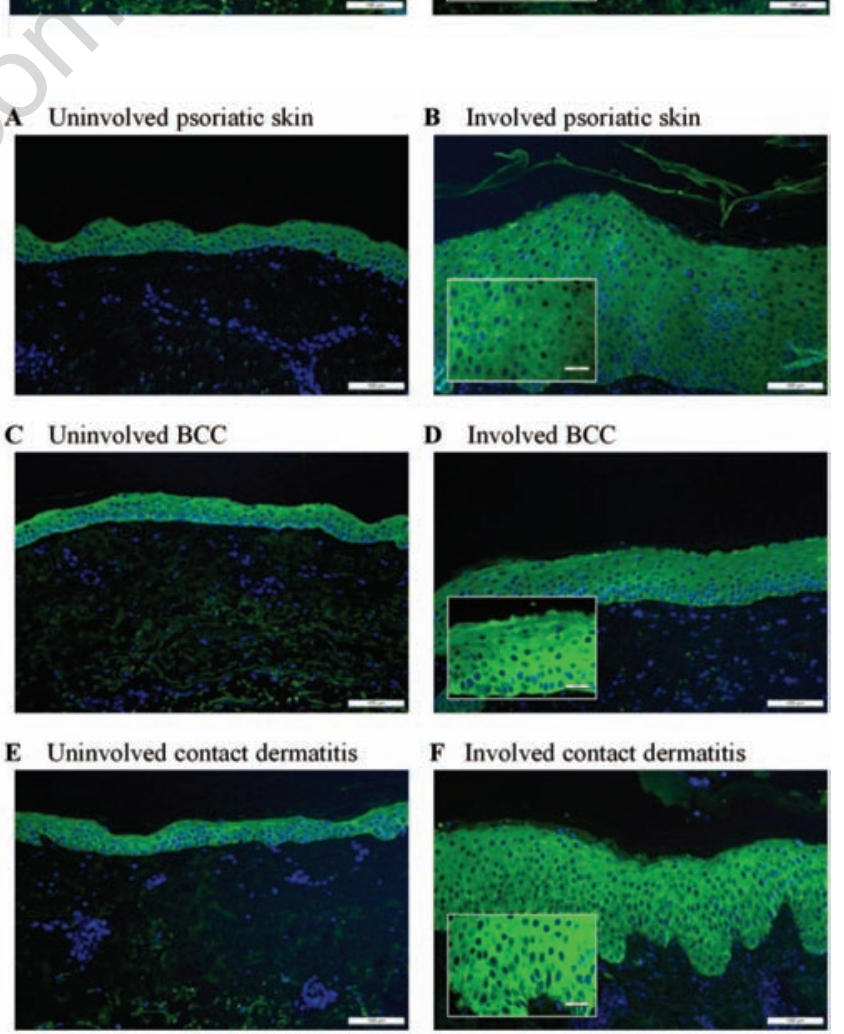

D Involved BCC

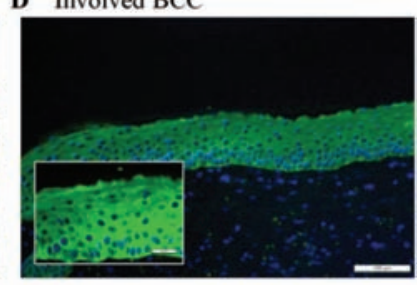

F Involved contact dermatitis

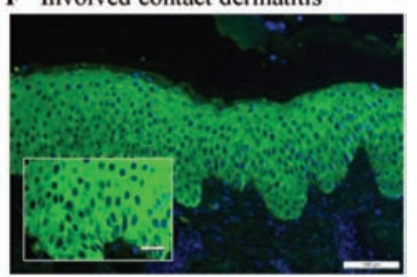

14-3-3 protein is involved in the regulation of transcription and translation by interacting with DNA/mRNA-binding proteins. Tristetraprolin (TTP) is a mRNA regulating protein, which through binding to AU-rich elements in mRNA (e.g. TNF- $\alpha$ ), induces mRNA aPKion and subsequently degradation. ${ }^{21}$ MAPK-activated protein kinase 2 (MK2) -induced phosphorylation of TTP creates a
Figure 3. Immuno fluorescence staining with 14-3-3 $\tau$. Localisation of 14 $3-3 \tau$ in the epidermis and dermis was analysed with immunofluorescence staining. Nuclear staining was performed using DAPI (blue). Green (Alexa Fluor 488) demonstrates $14-3-3 \tau$. The following paired tissue sections were stained: (A) uninvolved psoriatic skin, (B) involved psoriatic skin, (C) uninvolved $\mathrm{BCC}$, (D) involved BCC, (E) uninvolved allergic contact dermatitis, (F) involved allergic contact dermatitis.
Figure 4. Immuno fluorescence staining with 14-3-3 $\sigma$. Localisation of 14$3-3 \sigma$ in the epidermis and dermis was analysed with i m m un ofluores cence staining. Nuclear staining was performed using DAPI (blue). Green (Alexa Fluor 488) demonstrates 14-3$3 \sigma$. The following paired tissue sections were stained: (A) uninvolved psoriatic skin, (B) involved psoriatic skin, (C) uninvolved BCC, (D) involved BCC, (E) uninvolved allergic contact dermatitis, (F) involved allergic contact dermatitis. 
functional binding site for 14-3-3. When 14-3-3 binds to TTP, the mRNA-degrading capabilities of TTP is inhibited. ${ }^{22} \mathrm{~A}$ previous work from our group demonstrated increased MK2 activation in involved psoriatic skin compared with uninvolved skin. The protein expression of TNF- $\alpha$ was also elevated in psoriatic skin compared with uninvolved skin, whereas the TNF- $\alpha$ mRNA level was similar, indicating a posttranscriptional regulation of TNF- $\alpha$ expression in psoriasis and MK2 was suggested to play a role in this posttranscriptional regulation. ${ }^{23}$ It is possible that MK2 regulates TNF- $\alpha$ expression through TTP and 14-3-3. Own unpublished results showed no regulation of TTP mRNA and protein expression in involved psoriatic skin compared with uninvolved psoriatic skin (data not shown). Instead this study showed that the mRNA expression of six isoforms of 14-3-3 was elevated in involved psoriatic skin compared with uninvolved skin, but only 14-3$3 \tau$ was significantly elevated at the protein level. The precise role of $14-3-3 \tau$ in the regulation of TNF- $\alpha$ expression in psoriasis is not yet known. It is intriguing to speculate that an increased activity of MK2 combined with elevated protein expression of $14-3-3 \tau$ inhibits the destabilising effect of TTP on TNF- $\alpha$ mRNA thereby promoting TNF- $\alpha$ protein expression, but further studies are needed to clarify this.

Powell et al..$^{24}$ demonstrated that MK2 phophorylation of 14-3-3 $\zeta$ at Ser-58 compromises 14-3-3 dimerization. We found no regulation of $14-3-3 \zeta$ protein expression in involved psoriatic skin compared with uninvolved, but increased phosphorylation of 14-3$3 \zeta$ might affect its binding of TTP.

14-3-3 $\sigma$ regulates the G2/M cell cycle checkpoint and DNA damage induces $14-3-3 \sigma$ protein expression in a p53 dependent manner. ${ }^{10}$ Loss of 14-3-3 $\sigma$ increases the genomic instability in cells. Breast cancer cells lacking 14-3$3 \sigma$ expression shows an increased number of chromosomal breaks and gaps when exposed

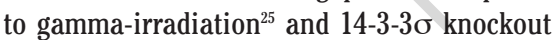
cells have an increased frequency of chromosome aberrations. ${ }^{26} 14-3-3 \sigma$ can be inactivated by CpG-metylation (epigenetic silencing) of the promoter region. ${ }^{25} \mathrm{CpG}$ methylation is a common event during carcinogenesis and has been reported in breast cancer, gastric, hepatocellular and small-cell carcinoma, oral squamous cell carcinoma, and basal cell carcinoma. ${ }^{9,27}$ Using immunohistochemistry Lodygin et al. ${ }^{11}$ observed a strongly reduced 14-3-3o expression in basal cell carcinomas compared with normal skin, whereas the 14-3-3o expression level in lesional psoriatic skin was comparable to normal skin. Interestingly, we found no regulation of 14-3-3 $\sigma$ mRNA expression in basal cell carcinomas, whereas an increased mRNA expression in involved psoriatic skin and in allergic contact dermatitis was

\begin{tabular}{|c|c|c|}
\hline Stimulus & $\begin{array}{l}\text { Period of } \\
\text { stimulation }\end{array}$ & $\begin{array}{l}\text { Response in the } 14-3-3 \tau \text { mRNA } \\
\text { level compared to vehicle }\end{array}$ \\
\hline TNF- $\alpha$ & $3 \mathrm{~h}, 6 \mathrm{~h}, 12 \mathrm{~h}$ and $24 \mathrm{~h}$ & No significant induction \\
\hline IFN- $\gamma$ & $3 \mathrm{~h}, 6 \mathrm{~h}, 12 \mathrm{~h}$ and $24 \mathrm{~h}$ & No significant induction \\
\hline IL-1 $\beta$ & $3 \mathrm{~h}, 6 \mathrm{~h}, 12 \mathrm{~h}$ and $24 \mathrm{~h}$ & No significant induction \\
\hline TPA & $0 \mathrm{~h}, 2 \mathrm{~h}, 4 \mathrm{~h}, 6 \mathrm{~h}$ and $12 \mathrm{~h}$ & No significant induction \\
\hline Anisomycin & $3 \mathrm{~h}, 6 \mathrm{~h}, 12 \mathrm{~h}$ and $24 \mathrm{~h}$ & No significant induction \\
\hline Calcium & $24 \mathrm{~h}$ and $48 \mathrm{~h}$ & $\begin{array}{l}\text { Tendency towards up regulation in } \\
\text { stimulated cells }\end{array}$ \\
\hline
\end{tabular}

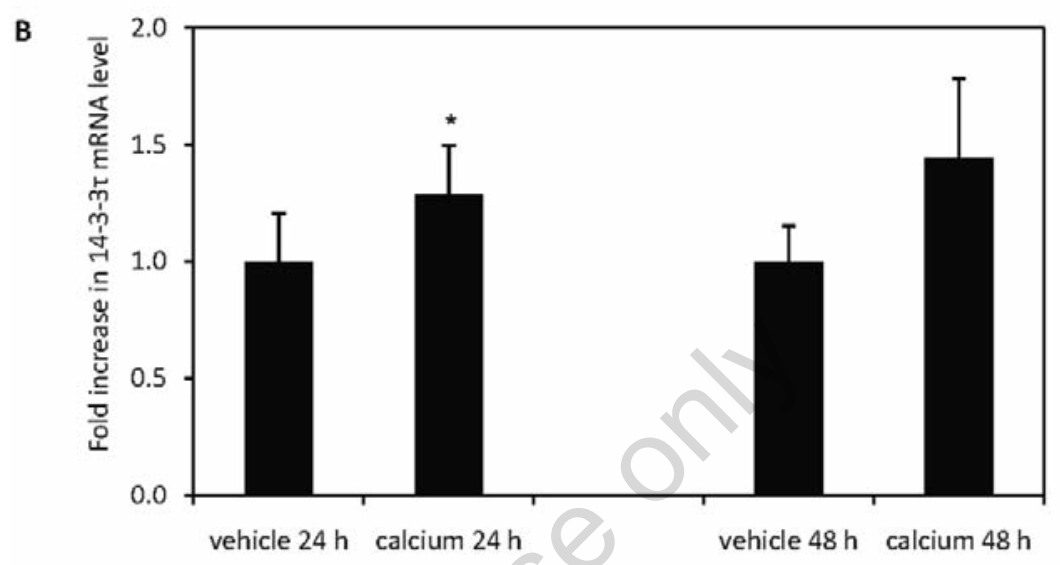

Figure 5. 14-3-3 $\tau$ mRNA expression in cultured normal human keratinocytes in vitro. Keratinocyte cultures were stimulated with a panel of different stimuli. mRNA expression was analysed by quantitative reverse transcription-polymerase chain reaction and the detected levels were normalized to the housekeeping gene RPLPO. Cell cultures incubated with vehicles were indexed to one. (A) Shows the different stimuli, incubation period and whether there was an induction of 14-3-3 $\tau$ mRNA expression or not. No significant induction was defined as no significant changes in the 14-3-3 $\tau$ mRNA level compared with vehicle. (B) Only incubation with calcium resulted in a significantly increased mRNA level of $14-3-3 \tau$ in the keratinocytes. Bars indicate mean $\pm S D$. ${ }^{*} P<0.05 . n=3$.

detected in this study. Furthermore, a slight although statistically insignificant increase in 14-3-3 $\sigma$ protein expression was seen in involved psoriatic skin compared with uninvolved skin. These differences may be explained by the different techniques used in the two studies. We detected mRNA using qRT-PCR whereas protein levels were detected by immunohistochemistry by Lodygin et al. ${ }^{11}$ Maintaining or even increasing the expression of 14-3-3 $\sigma$ in hyperproliferative skin disorders like psoriasis and allergic contact dermatitis may be a key control point in preventing malignant transformation by activating the G2/M checkpoint in the cell cycles.

Taken together, we showed an upregulation of 14-3-3 $\tau$ expression in psoriasis, allergic contact dermatitis and BCC whereas the expression of 14-3-3 $\sigma$ was increased only in psoriasis and allergic contact dermatitis. Thus, functional studies of the individual 14-3-3 isoforms in keratinocytes are highly needed in order to increase our understanding of a possible role of the various 14-3-3 isoforms in the pathogenesis of both benign and malignant skin diseases.

\section{References}

1. Martens GJ, Piosik PA, Danen EH. Evolutionary conservation of the 14-3-3 protein. Biochem Biophys Res Commun 1992;184:1456-9.

2. Aitken A. 14-3-3 proteins: a historic overview. Semin Cancer Biol 2006;16:16272.

3. Carlson FD. Physiological and biochemical aspects of nervous integration: a symposium held under the auspices of the Society of General Physiologists at its annual meeting at the Marine Biological Laboratory Woods Hole, Mass., Aug. 30Sept. 2, 1967. Englewood Cliffs, N.J.: 1968.

4. van Hemert MJ, Steensma HY, van Heusden GP. 14-3-3 proteins: key regulators of cell division, signalling and apoptosis. Bioessays 2001;23:936-46.

5. Boston PF, Jackson P, Thompson RJ. Human 14-3-3 protein: radioimmunoassay, tissue distribution, and cerebrospinal fluid levels in patients with neurological disor- 
ders. J Neurochem 1982;38:1475-82.

6. Pozuelo RM, Geraghty KM, Wong BH et al. 14-3-3-affinity purification of over 200 human phosphoproteins reveals new links to regulation of cellular metabolism, proliferation and trafficking. Biochem $\mathrm{J}$ 2004;379:395-408.

7. Bridges D, Moorhead GB. 14-3-3 proteins: a number of functions for a numbered protein. Sci STKE 2004;re10.

8. Ichimura $\mathrm{T}$, Isobe $\mathrm{T}$, Okuyama $\mathrm{T}$ et al. Molecular cloning of cDNA coding for brain-specific 14-3-3 protein, a protein kinase-dependent activator of tyrosine and tryptophan hydroxylases. Proc Natl Acad Sci USA 1988;85:7084-8.

9. Hermeking H. The 14-3-3 cancer connection. Nat Rev Cancer 2003;3:931-43.

10. Hermeking $\mathrm{H}$, Lengauer $\mathrm{C}$, Polyak $\mathrm{K}$ et al. 14-3-3 sigma is a p53-regulated inhibitor of G2/M progression. Mol Cell 1997;1:3-11.

11. Lodygin D, Yazdi AS, Sander CA, et al. Analysis of 14-3-3sigma expression in hyperproliferative skin diseases reveals selective loss associated with CpG-methylation in basal cell carcinoma. Oncogene 2003;22:5519-24.

12. Lau JM, Wu C, Muslin AJ. Differential role of 14-3-3 family members in Xenopus development. Dev Dyn 2006;235:1761-76.

13. Lau JM, Jin X, Ren J et al. The 14-3-3tau phosphoserine-binding protein is required for cardiomyocyte survival. Mol Cell Biol 2007;27:1455-66.

14. Danes CG, Wyszomierski SL, Lu J, et al. 14-
3-3 zeta down-regulates p53 in mammary epithelial cells and confers luminal filling. Cancer Res 2008;68:1760-7.

15. Takihara Y, Matsuda Y, Hara J. Role of the beta isoform of 14-3-3 proteins in cellular proliferation and oncogenic transformation. Carcinogenesis 2000;21:2073-7.

16. Qi W, Liu X, Chen W, et al. Overexpression of 14-3-3gamma causes polyploidization in H322 lung cancer cells. Mol Carcinog 2007;46:847-56.

17. Choi KC, Lee S, Kwak SY et al. Increased expression of 14-3-3varepsilon protein in intrinsically aged and photoaged human skin in vivo. Mech Ageing Dev 2005; 126:629-36.

18. Kilani RT, Medina A, Aitken A, et al. Identification of different isoforms of 14-33 protein family in human dermal and epidermal layers. Mol Cell Biochem 2008;314: 161-9.

19. Minang JT, Troye-Blomberg M, Lundeberg L, Ahlborg N. Nickel elicits concomitant and correlated in vitro production of Th1-, Th2-type and regulatory cytokines in subjects with contact allergy to nickel. Scand J Immunol 2005;62:289-96.

20. Kragballe K, Fallon JD. Increased aggregation and arachidonic acid transformation by psoriatic platelets: evidence that platelet-derived 12-hydroxy-eicosatetraenoic acid increases keratinocyte DNA synthesis in vitro. Arch Dermatol Res 1986;278:449-53.

21. Chrestensen CA, Schroeder MJ,
Shabanowitz J et al. MAPKAP kinase 2 phosphorylates tristetraprolin on in vivo sites including Ser178, a site required for 14-3-3 binding. J Biol Chem 2004;279: 10176-84

22. Stoecklin G, Stubbs T, Kedersha N et al. MK2-induced tristetraprolin:14-3-3 complexes prevent stress granule association and ARE-mRNA decay. EMBO J 2004;23: 1313-24.

23. Johansen C, Funding AT, Otkjaer $\mathrm{K}$ et al. Protein expression of TNF-alpha in psoriatic skin is regulated at a posttranscriptional level by MAPK-activated protein kinase 2. J Immunol 2006;176:1431-8.

24. Powell DW, Rane MJ, Joughin BA et al. Proteomic identification of 14-3-3zeta as a mitogen-activated protein kinase-activated protein kinase 2 substrate: role in dimer formation and ligand binding. Mol Cell Biol 2003;23:5376-87.

25. Ferguson AT, Evron E, Umbricht CB et al. High frequency of hypermethylation at the 14-3-3 sigma locus leads to gene silencing in breast cancer. Proc Natl Acad Sci USA 2000;97:6049-54.

26. Dhar S, Squire JA, Hande MP, et al. Inactivation of 14-3-3sigma influences telomere behavior and ionizing radiationinduced chromosomal instability. Mol Cell Biol 2000;20:7764-72.

27. Lodygin D, Hermeking $\mathrm{H}$. The role of epigenetic inactivation of 14-3-3sigma in human cancer. Cell Res 2005;15:237-46. 